Classification

Physics Abstracts

$06.50-07.80-78.70$

\title{
The parameterless correction method in X-ray microanalysis
}

\author{
Eric Van Cappellen \\ University of Antwerp, RUCA, Groenenborgerlaan 171, B-2020 Antwerp, Belgium
}

(Received December 15, 1989; accepted January 7, 1990)

\begin{abstract}
Résumé. - Une méthode permettant de corriger les effets d'absorption et de fluorescence en microanalyse $\mathrm{X}$ d'échantillons transparents pour la microscopie en transmission (S.) T.E.M. est présentée. Cette procédure n'exigeant la connaissance d'aucun paramètre comme l'épaisseur ou la densité de l'echantillon ni d'aucun coefficient comme les coefficients d'absorption de masse ou encore des coefficients de rendement de fluorescence, est extrêmement utile et pratique pour effectuer des analyses de routine d'échantillons minces et a été appelé : " parameterless correction method". De surcroît tout effet dépendant de l'épaisseur de l'échantillon, comme la présence de couches de surface ou l'effet d'orientation crystallographique (l'effet Borrmann), peuvent-être détectés par cette méthode.
\end{abstract}

\begin{abstract}
The parameterless correction method to perform absorption and fluorescence corrections in X-ray microanalysis of tranparent (S.) T.E.M. specimens is presented and supported. This correction procedure requires no external parameters such as foil thickness or density, and no coefficients such as mass absorption coefficients or fluorescence yield coefficents and is therefore suitable for fast routine thin film microanalysis. Furthermore, thickness dependent artefacts such as surface and contamination layers and crystallographic orientation effects (the Borrmann effect) can be detected by the method.
\end{abstract}

\section{Introduction.}

It is now well established that even in thin film X-ray microanalysis absorption and sometimes fluorescence corrections are needed to acquire accurate quantitative data. In the plane parallel foil model an absorption correction already requires three external parameters : the foil thickness, the density and the take-off angle of the X-rays. The classical fluorescence corrections of Tixier $[1,2]$ and Nockolds [3] all derived for a plane parallel foil, require even more parameters and are therefore generally omitted. Moreover the models strongly disagree on the correction amplitudes and the hypothetical plane parallel shape is seldomly encountered in experimental situations.

The secondary or fluorescence emission of a spectral line can always be expanded in terms of $T$, the mass thickness of the specimen defined along the optical axis of the microscope. Computer simulations of this secondary radiation in wedge shaped specimens revealed that this polynomial in $T$ without constant term, is smooth and that the first or linear term is directly proportional 
with the wedge angle $\alpha[4,5]$. To evaluate this influence of the specimen shape on the secondary emission, simulations for a $\mathrm{Cu}-50$ at $\% \mathrm{Cr}$ alloy are shown in figure 1 for different wedge angles $\left(\alpha=0^{\circ}, 10^{\circ}, 20^{\circ}, 30^{\circ}\right.$ and $\left.40^{\circ}\right)$. This graph shows the dramatic increase in fluorescence emission with increasing wedge angle. For a thickness of $50 \mathrm{~nm}$, a thickness very common in transmission or scanning transmission electron microscopy the secondary emission is twice as important for a wedge of $10^{\circ}$ as for a plane parallel foil whereas for a wedge of $40^{\circ}$ this intensity is already six times as large.

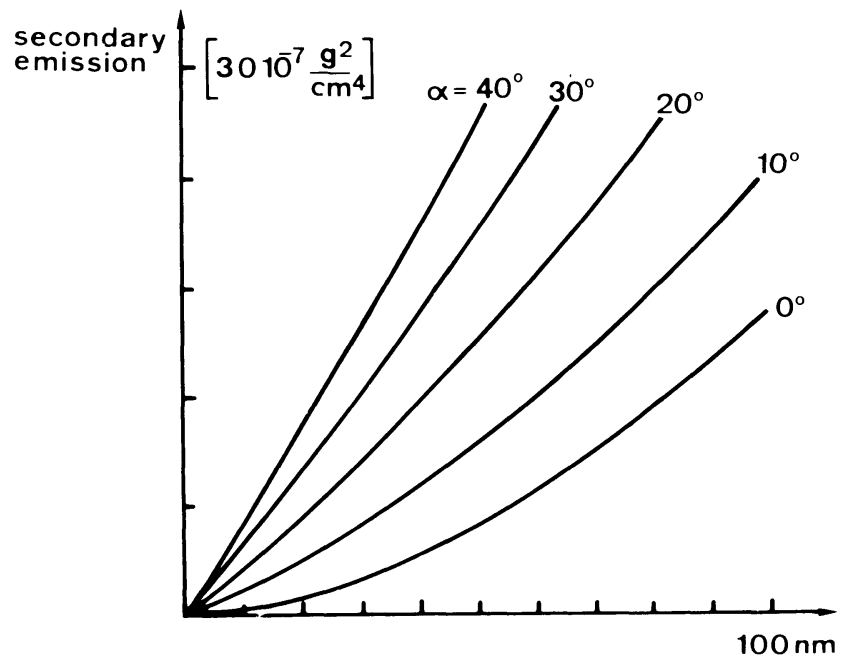

Fig. 1. - Secondary or fluorescence radiation in wedge shaped targets (Cu-50at\% Cr) [4]. The slope of the curves in the origin $(0,0)$ is directly proportional with the wedge angle $\alpha$.

In other words, a very modest change in the shape of the specimen can have a large influence on the secondary emission. This confirms our conviction that no fluorescence correction can be entirely satisfactory unless the exact shape of the specimen is introduced in the correction procedure.

Since X-rays can propagate considerable distances in the specimen when compared with the typical dimensions of the primary excitation volume, the secondary excitation volume will be some orders of magnitude larger than the primary one. As a consequence the specimen shape should not only be known in the neighbourhood of the electron irradiated area but in a much larger area, typically some tenths of a millimeter or even more in diameter. Even if a fluorescence correction procedure based on an on-line computer simulation for an arbitrary target geometry would be developed in the future it remains extremely doubtful whether it will ever be feasible to determine the exact specimen shape over such a large area for a routine analysis.

To overcome the practical problems enumerated above an alternative correction procedure has been developed capable of correcting for absorption and for fluorescence to a large extend and was called the parameterless correction method [6-8]. 


\section{Principle of the parameterless correction method.}

2.1 Methodology. - The name "parameterless correction method" is proposed because no external parameters are needed during execution. This implies other concessions. The major one is that not one but several spectra are needed for one analysis. The spectra are taken at different sites on the specimen with different thickness. It is obvious that the specimen must have a homogeneous composition in the analysed area which in practice must be about $1 \times 0.1 \mu \mathrm{m}$ in size.

A characteristic line of an energy dispersive X-ray spectrum can always be considered as being composed of primary and secondary characteristic radiation both attenuated due to absorption in the specimen. Both components can be expressed as polynomials in $T$ the thickness or mass thickness of the foil. The intensity of a characteristic line vanishes monotonically at zero thickness, consequently it's polynomial in $T$ will have no constant term. Hence the ratio of two net peak integrals, as encountered in the Cliff Lorimer [9] equation is a polynomial in the thickness with a constant term. This constant term times the proper $k$-factor is the mass concentration ratio of the considered elements.

From the different spectra taken at different thicknesses uncorrected mass concentration ratios are calculated with the Cliff Lorimer technique. These results are plotted versus the foil thickness and a least square fit is used to extrapolate the mass concentration ratio to zero thickness. As will be demonstrated lateron this extrapolated value is free from absorption and major fluorescence effects.

In order to make the procedure "parameterless" the foil thickness or mass thickness used for the extrapolation must be substituted by an internal measure. This substitute henceforth termed "internal thickness measure" must monotonically tend to zero for vanishing specimen thickness so as to obtain the same extrapolation value as with the true thickness.

We shall now discuss two possible candidates for this internal measure.

\subsection{INTERNAL THICKNESS MEASURE.}

2.2.1 Bremsstrahlung. - In the quantitative method for biological tissues proposed by Hall [10] the X-ray intensity in a background window is used as indirect measure for the specimen thickness. At first sight a Bremsstrahlung band, free from characteristic radiation, is indeed a good substitute measure for the foil thickness, but as will be experimentally demonstrated, not all white radiation originates in the specimen. As a consequence Bremsstrahlung does not totally vanish for a hypothetical zero thickness specimen and must therefore be rejected as internal thickness measure.

2.2.2 Characteristic radiation. - As already mentioned, characteristic radiation can be expressed as a smooth monotone polynomial in the thickness without a constant term. Consequently the foil thickness can be expanded as the inverse smooth monotone polynomial in the intensity of the peak of the considered element without a constant term, on condition that this element is only present in the specimen. A net peak integral or a sum of net peak integrals will prove to be an excellent internal thickness measure.

\section{Mathematical formulation.}

Two slightly different but equivalent formulations of the parameterless correction method will be presented. The first one is straightforward and starts from the assumption that a characteristic line of an element $X$ consists of primary and secondary radiation both corrected for absorption. 
The second approach starts from the Cliff-Lorimer equation extended with an absorption and a fluorescence correction. Both approaches lead to the same conclusions since the absorption and fluorescence corrections of the Cliff-Lorimer equation are transformed into expressions containing the primary and secondary emission of the considered elements.

\subsection{FIRST APPROACH.}

3.1.1 The intensity $I_{X}$ of a characteristic peak of an element $X$. - The primary emission of an element $X$, corrected for absorption in the direction of the detector can be approximated as follows (e.g. $[1,2])$ :

$$
I_{X}^{\mathrm{P}}=C C_{X} \int_{0}^{T} \varphi(t) \exp \left(-\mu_{X} s(t)\right) \mathrm{d} t
$$

All distances are expressed in mass-length units and $\varphi(t)$ is the mass-depth ionization distribution function along the optical axis. The total mass thickness of the foil along this optical axis is denoted $T . C_{X}$ and $\mu_{X}$ are respectively the mass concentration and the mass absorption coefficient of element $X$ whereas $s(t)$ is the distance the primary X-ray photons must travel in the specimen in the direction of the detector before leaving the surface. This function strongly depends on the shape of the specimen and the detection direction. The constant $C$ takes account of the fluorescence yield of the excited shell of element $X$, the weight factor of the line under consideration, the fraction of the isotropic primary emission emitted towards the detector and last but not least the detector efficiency for the considered line. Equation (1) can always be expanded in a power scries in $T$ :

$$
I_{X}^{\mathrm{P}}=\sum_{i=1}^{\infty} p_{i}^{X} T^{i}
$$

It should be noted that there is no constant term and that for an infinitesimally thin film the first (linear) term will be dominant.

The secondary emission $I_{X}^{\mathrm{F}}$ of an element $X$, corrected for absorption in the direction of the detector can be approximated as follows $[4,5]$ :

$$
I_{X}^{\mathrm{F}}=C^{\prime} \int_{0}^{T} \int_{\text {spec. }} \varphi(t) \frac{\exp \left(-\mu_{Y}|\mathbf{r}|\right)}{|\mathbf{r}|^{2}} \exp \left(-\mu_{X} \cdot q(\mathbf{r}, t)\right) \mathrm{d} V \mathrm{~d} t
$$

This equation is a four dimensional integral (three dimensional over the specimen volume $(\mathrm{d} V)$ and one dimensional over the optical axis $(\mathrm{d} t)$ ). The vector $\mathbf{r}$ connects the site where the primary emission of an element $Y$ is emitted with the site where secondary emission of element $X$ is generated by the primary radiation of element $Y . q(\mathbf{r}, t)$ is analogously the distance to be bridged by the fluorescence radiation before leaving the surface in the direction of the detector. The constant $C^{\prime}$ now mainly contains the fluorescence yields of the shells of elements $X$ and $Y$ and their respective weight factors, the ionisation yield of element $X$, the mass absorption coefficient of element $Y$ in pure $X$, the mass concentrations of the elements $X$ and $Y$.

Equation (3) can also be expanded in a power series in $T$ and in the case of wedge shaped specimens, computer simulations showed that the linear term is proportional to the wedge angle [5].

$$
I_{X}^{\mathrm{F}}=\sum_{i=1}^{\infty} f_{i}^{X} T^{i}
$$

The total intensity $I_{X}$ of element $X$ is given by the sum of (2) and (4) :

$$
I_{X}=I_{X}^{\mathrm{P}}+I_{X}^{\mathrm{F}}=\sum_{i=1}^{\infty} a_{i}^{X} T^{i}
$$


and :

$$
a_{i}^{X}=p_{i}^{X}+f_{i}^{X} \forall i
$$

3.1.2 The mass thickness T. - Equation (5) can be inversed so as to yield the mass thickness $T$ :

$$
T=\sum_{i=1}^{\infty} b_{i}^{X} I_{X}^{i}
$$

Note that all the coefficients $p_{i}^{X}, f_{i}^{X}, a_{i}^{X}$ and $b_{i}^{X}$ strongly depend on the shape of the specimen and the detection geometry.

3.1.3 The intensity ratio of two elements $A$ and $B$. - As usual for a thin film ratio technique the ratio of two peak intensities of elements $A$ and $B$ is considered :

$$
\frac{I_{A}}{I_{B}}=\sum_{i=0}^{\infty} A_{i}^{A B} T^{i}
$$

The mass thickness $T$ can be replaced by (7):

$$
\frac{I_{A}}{I_{B}}=\sum_{i=0}^{\infty} B_{i}^{A B} I_{X}^{i}
$$

The constant terms $A_{0}^{A B}$ and $B_{0}^{A B}$ are equal to the ratio $I_{A} / I_{B}$ for mass thickness tending to zero and read :

$$
A_{0}^{A B}=B_{0}^{A B}=\frac{a_{1}^{A}}{a_{1}^{B}}=\frac{p_{1}^{A}+f_{1}^{A}}{p_{1}^{B}+f_{1}^{B}}
$$

The intensity ratio $I_{A} / I_{B}$ can be converted into a mass concentration ratio when divided by the Cliff-Lorimer $k_{A B}$-factor. This mass concentration ratio depends on $T$ (or $I_{X}$ ) and is therefore called the "uncorrected mass concentration ratio" and is denoted $\left(C_{A} / C_{B}\right)^{\prime}$. This ratio tends to a fixed value as a limit when $T$ or $I_{X}$ approaches zero:

$$
\lim _{T, I_{X} \rightarrow 0}\left(\frac{C_{A}}{C_{B}}\right)^{\prime}=\frac{1}{k_{A B}} \cdot \frac{p_{1}^{A}+f_{1}^{A}}{p_{1}^{B}+f_{1}^{B}}
$$

On the other hand, the exact mass concentration ratio $C_{A} / C_{B}$ can be derived from the CliffLorimer equation on condition that no absorption and, as a consequence, no fluorescence of $\mathrm{X}$-rays occur in the specimen. This condition, fulfilled in an infinitesimal thin plane parallel foil, reads :

$$
\frac{C_{A}}{C_{B}}=\frac{1}{k_{A B}} \lim _{T \rightarrow 0}\left(\frac{I_{A}^{\mathrm{P}}}{I_{B}^{\mathrm{P}}}\right)=\frac{1}{k_{A B}} \frac{p_{1}^{A}}{p_{1}^{B}}
$$

From equations (11) and (12) it is clear that strictly speaking the extrapolated value of the uncorrected mass concentration ratio (11) is not equal to the exact ratio (12). However the coefficients $p_{1}^{X}$ and $f_{1}^{X}$ represent respectively the primary and the secondary emission of an element $X$ when $T$ the specimen mass thickness (or $I_{X}$ ) approaches zero. It is therefore beyond doubt that $f_{1}^{X}$ is much smaller than $p_{1}^{X}$ or :

$$
f_{1}^{A} / p_{1}^{A}<<1 \text { and } f_{1}^{B} / p_{1}^{B}<<1
$$


This assumption will be explicitly demonstrated in paragraph 4. Consequently in the limit, for $T$ or $I_{X}$ approaching zero, one has :

$$
\lim _{T, I_{X} \rightarrow 0}\left(C_{A} / C_{B}\right)^{\prime}=C_{A} / C_{B}
$$

This last statement proves the parameterless correction method.

In practice, uncorrected mass concentration ratios are measured at different sites with different mass thickness and are plotted as a function of a net peak integral $I_{X}$. By means of a least square fit through these experimental points the extrapolation to $I_{X}=0$ is obtained. Equation (14) states that this extrapolated value is equal to the exact mass concentration ratio $C_{A} / C_{B}$.

- Remarks.

The net peak integral $I_{X}$ used for the extrapolation can be replaced by a sum of net peak integrals. The only condition is that it should be characteristic radiation. Obviously the peaks $I_{A}$ and $I_{B}$ can also be used.

It has been shown that for a perfect plane parallel foil the linear term of fluorescence emission equals zero $\left(f_{1}=0\right)[5]$. Hence $f_{1}^{A}=f_{1}^{B}=0$ so that from (11) and (12) it is clear that for $T$ or $I_{X}$ approaching zero $\left(C_{A} / C_{B}\right)^{\prime}=\left(C_{A} / C_{B}\right)$ without any assumption.

The equations for primary (1) and secondary (3) emission are derived by assuming a one dimensional excitation volume along the optical axis. Actually the one dimensional integration along the optical axis from 0 to $T$ should be replaced by a three dimensional integration over the real excitation volume. However, this would not affect the conclusions of the parameterless correction method since $T$ is then simply replaced by $V$ the real excitation volume. Equation (5) then becomes :

$$
I_{X}=\sum_{i=1}^{\infty} a_{i}^{\prime X} V^{i}
$$

and consequently :

$$
V=\sum_{i=1}^{\infty} b_{i}^{\prime X} I_{X}^{i}
$$

With equations (15) and (16) a completely analogous derivation is possible as with equations (3) and (7). The intensity of two elements $A$ and $B$ now reads :

$$
\frac{I_{A}}{I_{B}}=\sum_{i=0}^{\infty} A_{i}^{\prime A B} V^{i}=\sum_{i=0}^{\infty} B^{\prime A B} I_{X}^{i}
$$

and :

$$
A_{0}^{\prime A B}=B_{0}^{\prime A B}=\frac{a_{1}^{\prime A}}{a_{1}^{\prime B}}=\frac{a_{1}^{A}}{a_{1}^{B}}=B_{0}^{A B}=A_{0}^{A B}
$$

All the conclusions drawn in the previous paragraph remain valid. The reason why the initial reasoning was carried out with the mass thickness $T$ instead of the excitation volume $V$ is that in transmission or scanning transmission electron microscopy the notion mass thickness is more common than the notion excitation volume. It is indeed easier to claim that two spectra were taken at different (mass) thicknesses than with different excitation volumes. The mass thickness of an area can be directly associated with the transparancy of it while by no means the excitation volume (size and intensity) can be visualized. 
3.2 SECOND APPROACH. - The second more classical approach starts from the Cliff Lorimer equation but is entirely equivalent with the first one since the same equations for primary and secondary emission will be used. The corrected Cliff Lorimer equation reads [9] :

$$
\frac{C_{A}}{C_{B}}=k_{A B}^{-1} \frac{I_{A}}{I_{B}}(A \cdot F)^{-1}
$$

$I_{A}$ and $I_{B}$ are the measured intensities and $A$ and $F$ are respectively the absorption and fluorescence correction factors. The classical notation $f(\chi)(\chi=\mu \cdot \operatorname{cosec} \theta)$ for the absorption correction of a peak will not be used here since strictly speaking it is only valid for a plane parallel foil, and it could lead to confusion with fluorescence correction terms. " $a_{X}$ " and " $f_{X}$ " respectively denote the absorption and fluorescence corrections of the peak of element $X$ and are defined as follows :

$$
a_{X}=\frac{I_{X}^{\mathrm{P}}}{I_{X}^{0}}
$$

and

$$
f_{X}=1+\frac{I_{X}^{\mathrm{F}}}{I_{X}^{\mathrm{P}}}
$$

$I_{X}^{0}$ is the total primary emission of a specific line of element $X$ generated in the specimen. The definitions (20) and (21) can be transformed with equations (1) and (3) :

$$
\begin{gathered}
a_{X}=\frac{\int_{0}^{T} \varphi(t) \exp \left(-\mu_{X} s(t)\right) \mathrm{d} t}{\int_{0}^{T} \varphi(t) \mathrm{d} t} \\
f_{X}=1+\frac{C^{\prime} \int_{0}^{T} \int_{\text {spec }} \varphi(t) \frac{\exp \left(-\mu_{Y}|\mathbf{r}|\right)}{|\mathbf{r}|^{2}} \exp \left(-\mu_{X} q(\mathbf{r}, t)\right) \mathrm{d} V \mathrm{~d} t}{C_{0} \int \varphi(t) \exp \left(-\mu_{X} s(t)\right) \mathrm{d} t}
\end{gathered}
$$

Equations (22) and (23) can be expanded in power series in $T$ :

$$
\begin{gathered}
a_{X}=\sum_{i=0}^{\infty} m_{i}^{X} T^{i} \quad \text { with } m_{0}=1 \\
f_{X}=1+\sum_{i=0}^{\infty} \ell_{i}^{X} T^{i}
\end{gathered}
$$

The respective ratios for two elements $A$ and $B$ read :

$$
\begin{gathered}
\frac{a_{A}}{a_{B}}=\sum_{i=0}^{\infty} M_{i}^{A B} T^{i} \text { with } M_{0}^{A B}=1 \\
\frac{f_{A}}{f_{B}}=\sum_{i=0}^{\infty} L_{i}^{A B} T^{i}
\end{gathered}
$$

Using (26) and (27) the general correction factor of the Cliff Lorimer equation becomes :

$$
A \cdot F=\sum_{i=0}^{\infty} C_{i}^{A B} T^{i}
$$


where :

$$
C_{0}^{A B}=L_{0}^{A B}=\frac{1+\ell_{1}^{A}}{1+\ell_{1}^{B}}=\frac{1+f_{1}^{A} / p_{1}^{A}}{1+f_{1}^{B} / p_{1}^{B}}
$$

Note that $\ell_{1}^{X}=f_{1}^{X} / p_{1}^{X}$ when referring to the polynomials (2) and (4). $\left(C_{A} / C_{B}\right)^{\prime}$ denotes again the uncorrected mass concentration ratio and is equal to the uncorrected Cliff Lorimer equation :

$$
\left(\frac{C_{A}}{C_{B}}\right)^{\prime}=k_{A B}^{-1} \frac{I_{A}}{I_{B}}
$$

This can be transformed to :

$$
\left(\frac{C_{A}}{C_{B}}\right)^{\prime}=\frac{C_{A}}{C_{B}} \cdot A \cdot F=\frac{C_{A}}{C_{B}} \sum_{i=0}^{\infty} G_{i}^{A B} T^{i}
$$

This polynomial can again be transformed into a polynomial in $I_{X}$ with coefficients $G_{i}^{A B}$, but obviously $G_{0}^{A B}=G_{0}^{A B}$.

The limit, as $T$ or $I_{X}$ approaches zero, of $\left(C_{A} / C_{B}\right)^{\prime}$ equals :

$$
\lim _{T, I_{X} \rightarrow 0}\left(\frac{C_{A}}{C_{A}}\right)^{\prime}=\frac{C_{A}}{C_{B}} G_{0}^{A B}=\frac{C_{A}}{C_{B}} \frac{1+f_{1}^{A} / p_{1}^{A}}{1+f_{1}^{B} / p_{1}^{B}}
$$

If approximation (13) is valid, equation (32) again states that the extrapolation value of the uncorrected mass concentration ratio approaches the exact ratio and since :

$$
\frac{C_{A}}{C_{B}}=\frac{1}{k_{A B}} \frac{I_{A}^{0}}{I_{B}^{0}}=\frac{1}{k_{A B}} \lim _{T \rightarrow 0}\left(\frac{I_{A}^{\mathrm{P}}}{I_{B}^{\mathrm{P}}}\right)=\frac{1}{k_{A B}} \frac{p_{1}^{A}}{p_{1}^{B}}
$$

equation (32) equals (11). The parameterless correction method depends on the validity of condition (13) which is also equivalent with :

$$
\lim _{T, I_{X} \rightarrow 0}\left(\frac{I_{X}^{\mathrm{F}}}{I_{X}^{\mathrm{P}}}\right)<<1
$$

This condition is examined in the next paragraph.

4. Systematic error estimation : $\lim _{T \rightarrow 0}\left(I_{X}^{\mathrm{F}} / I_{X}^{\mathrm{P}}\right)$.

The systematic extrapolation error governed by condition (34) will now be examined. Expressions for the primary $I_{X}^{\mathrm{P}}$ and secondary $I_{X}^{\mathrm{F}}$ emissions of an element $X$ are obtained for a small mass thickness $T$, and are evaluated using computer simulations of secondary emission in wedge shaped targets. 
4.1 THE PRIMARY EMISSION $I_{X}^{\mathrm{P}}$ FOR SMALL $T$. - The number of ionizations of the $j$-shell of element $X$ in an infinitesimal thin film is given by the formula of Castaing [2] :

$$
\mathrm{d} n_{X}^{j}=C_{X} \frac{N}{A_{X}} \psi_{X}^{j}(E) \mathrm{d} t
$$

where $C_{X}$ and $A_{X}$ are respectively the mass concentration and the atomic weight of element $X$. $\mathrm{d} t$ is the mass thickness of the foil and $\psi_{X}^{j}(E)$ is the ionization cross section for the $j$-shell of element $X$. The primary X-ray intensity of line $\delta, \mathrm{d} I_{X}^{\delta}$ (line $\delta$ is a characteristic line of the $j$-shell of element $X$ ) is equal to the number of ionizations (35) multiplied by the fluorescence yield $\omega_{X}^{j}$ of the $j$-shell and the weight factor $W_{X}^{\delta}$ of the considered line $\delta$ :

$$
\mathrm{d} I_{X}^{\delta}=W_{X}^{\delta} \omega_{X}^{j} \mathrm{~d} n_{X}^{j}
$$

Since an infinitesimal thin foil is considered, $E$ the energy of the exciting electrons can be replaced by $E_{0}$, the accelerating potential. Furthermore no absorption effects occur, in other words the detected primary emission is equal to the generated intensity multiplied by the fraction emitted towards the detector. If the solid angle spanned by the detector is denoted $\mathrm{d} \Omega^{\prime}, I_{X}^{\mathrm{P}}$ equals :

$$
I_{X}^{\mathrm{P}}=\frac{\mathrm{d} \Omega^{\prime}}{4 \pi} I_{X}^{0}=\frac{\mathrm{d} \Omega^{\prime}}{4 \pi} W_{X}^{\delta} \omega_{X}^{j} \frac{N}{A_{X}} \psi_{X}^{j}\left(E_{0}\right) C_{X} T
$$

4.2 THE SECONDARY EMISSION $I_{X}^{\mathrm{F}}$ FOR SMALL $T$. - To make an evaluation possible $I_{X}^{\mathrm{F}}$ will be derived for a wedge shaped specimen. In the final equation numerical data acquired from computer simulations will be used [5].

Consider an infinite wedge with a wedge angle $\alpha$. Primary radiation of an element $Y$ can excite element $X$ and generate secondary radiation of element $X$.

If only the tip of the wedge is irradiated by the electron beam (Fig. 2). The primary radiation of element $Y$ generated in the tip of the wedge reads (see (37)) :

$$
I_{Y}^{0}=W_{Y}^{\gamma} \omega_{Y}^{i} \frac{N}{A_{Y}} \psi_{Y}^{i}\left(E_{0}\right) C_{Y} T
$$

The fraction of this radiation reaching the element of volume $\mathrm{d} V$ in the wedge equals :

$$
\mathrm{d} I_{Y}=\frac{\mathrm{d} \Omega}{4 \pi} I_{Y}^{0} \exp \left(-\mu_{Y}|\mathbf{r}|\right)
$$

where $\mathrm{d} \Omega$ is the solid angle spanned by the element of volume $\mathrm{d} V$ and $\mu_{Y}$ the mass absorption coefficient of the $\gamma$-line of element $Y$. In $\mathrm{d} V$ the $\mathrm{X}$-atoms will absorb a certain primary intensity of element $Y$ equal to :

$$
\mathrm{d} I_{Y}^{X}=\mathrm{d} I_{Y} C_{X} \mu_{Y}^{X} \mathrm{~d}|\mathbf{r}|
$$

$\mu_{Y}^{X}$ is the mass absorption coefficient of the $\gamma$-line in a target consisting of pure $X$ atoms and d|r| is the distance travelled by the primary $Y$-radiation in $\mathrm{d} V$. The secondary radiation of element $X$ generated in $\mathrm{d} V$ and emitted towards the detector reads :

$$
\mathrm{d} I_{X}^{\mathrm{F}}=\frac{\mathrm{d} \Omega^{\prime}}{4 \pi} \mathrm{d} I_{Y}^{X} \frac{r_{X}^{j}-1}{r_{X}^{j}} W_{X}^{\delta} \omega_{X}^{j} \exp \left(-\mu_{X} \cdot q(\mathbf{r})\right)
$$




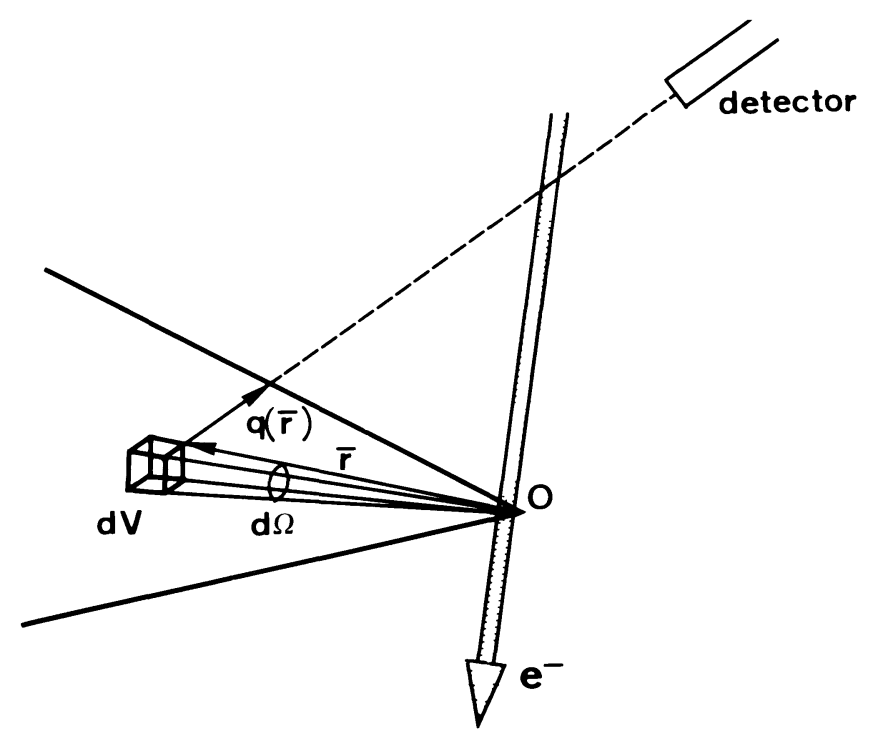

Fig. 2. - Model used to calculate the secondary emission when only the tip of the wedge is irradiated.

$\left(r_{X}-1\right) / r_{X}$ is the ionization yield of the $j$-shell of element $X$ and $\omega_{X}^{j}$ is the fluorescence yield of the same shell. $W_{X}^{\delta}$ is the weight factor of the $\delta$-line in the set of all $j$-shell ionizations and $\exp \left(-\mu_{X} q(\mathbf{r})\right)$ takes account of absorption of the secondary $\delta$-line emission in the direction of the detector.

The solid angle spanned by the detector is denoted $\mathrm{d} \Omega^{\prime}$. The total fluorescence contribution is obtained by an integration of $\mathrm{d} V$ over the whole specimen volume :

$$
I_{X}^{\mathrm{F}}=\int_{\text {spec. }} \mathrm{d} I_{X}^{\mathrm{F}}
$$

or :

$$
I_{X}^{\mathrm{F}}=\frac{\mathrm{d} \Omega^{\prime}}{16 \pi^{2}} \frac{r_{X}^{j}-1}{r_{X}^{j}} W_{X}^{\delta} \omega_{X}^{j} C_{X} \mu_{Y}^{X} I_{Y}^{0} \int_{\text {wedge }} \frac{\exp \left(-\mu_{Y}|\mathbf{r}|\right)}{|\mathbf{r}|^{2}} \exp \left(-\mu_{X} q(\mathbf{r})\right) \cdot \mathrm{d} V
$$

4.3 THE RATIO " $I_{X}^{\mathrm{F}} / I_{X}^{\mathrm{P}}$ " FOR SMALl $T$. - The ratio $I_{X}^{\mathrm{F}} / I_{X}^{\mathrm{P}}$ for small $T$ reads (Eq. (43) divided by Eq. (37)) :

$$
\begin{aligned}
\frac{I_{X}^{\mathrm{F}}}{I_{X}^{\mathrm{P}}} & =\frac{1}{4 \pi} \frac{r_{X}^{j}-1}{r_{X}^{j}} W_{Y}^{j} \omega_{Y}^{i} \mu_{Y}^{X} \frac{A_{X}}{A_{Y}} \frac{\psi_{Y}^{i}\left(E_{0}\right)}{\psi_{X}^{j}\left(E_{0}\right)} C_{Y} \\
& \times \int_{\text {wedge }} \frac{\exp \left(-\mu_{Y}|\mathbf{r}|\right)}{|\mathbf{r}|^{2}} \exp \left(-\mu_{X} q(\mathbf{r})\right) \mathrm{d} V
\end{aligned}
$$

Since this ratio is independent of $T$ it equals the limit as $T$ approaches zero. To evaluate the magnitude of equation (44) numerical data acquired with computer simulations of fluorescence radiation in wedge shaped targets will be used [5]. For small $T$ it can be shown that the four dimensional integral used for the simulations is equivalent to the three dimensional integral (44) 
multiplied by the mass thickness $T$. In other words the three dimensional integral (44) is the linear coefficient of the fluorescence polynomial in $T$. It was demonstrated that this coefficient is proportional to the wedge angle $\alpha$ and the proportionality factor will be denoted $K_{X Y}$ :

$$
K_{X Y} \alpha=\int_{\text {wedge }} \frac{\exp \left(-\mu_{Y}|\mathbf{r}|\right)}{|\mathbf{r}|^{2}} \exp \left(-\mu_{X} q(\mathbf{r})\right) \mathrm{d} V
$$

$K_{X Y}$ depends on the mass absorption coefficients $\mu_{X}$ and $\mu_{Y}$ and of the geometry of the specimen. It was also shown that $K_{X Y}$ is quite insensitive to the specimen orientation in the electron beam and to the $\mathrm{X}$-ray detection direction.

Equation (44) is the fluorescence to primary intensity ratio of the $\delta$-line of element $X$, in which only the secondary emission generated by the $\gamma$-line belonging to the $i$-shell ionization of element $Y$ is considered. I fact all the lines belonging to the $i$-shell ionizations have to be considered since they all contribute to the fluorescence emission of element $X$. All these lines are very close in energy, consequently they all have approximately the same mass absorption coefficient and the same ionization cross section. Furthermore the sum of all the weight factors of the lines of a set equals one $\left(\sum_{\text {set }} W_{Y}^{\gamma}=1\right)$.

The secondary to primary intensity ratio of element $X$ taking into account all the lines of the $i$ shell ionizations of element $Y$ as possible primary $\mathrm{X}$-ray excitation sources of the $X$ atoms then reads :

$$
\lim _{T \rightarrow 0}\left(\frac{I_{X}^{\mathrm{F}}}{I_{X}^{\mathrm{P}}}\right)=\frac{1}{4 \pi} \frac{r_{X}^{j}-1}{r_{X}^{j}} \omega_{Y}^{i} \mu_{Y}^{X} \frac{A_{X}}{A_{Y}} \frac{\psi_{Y}^{i}\left(E_{0}\right)}{\psi_{X}^{j}\left(E_{0}\right)} C_{Y} K_{X Y} \alpha
$$

The ionization cross section ratio $\psi_{Y}^{i}\left(E_{0}\right) / \psi_{X}^{j}\left(E_{0}\right)$ can be approximated with the BETHE formula [11]:

$$
\frac{\psi_{Y}^{i}\left(E_{0}\right)}{\psi_{X}^{j}\left(E_{0}\right)}=\frac{E_{X}^{j}}{E_{Y}^{i}} \frac{Z_{Y}^{i}}{Z_{X}^{j}} \frac{\ln \left(E_{0} / E_{Y}^{i}\right)}{\ln \left(E_{0} / E_{X}^{j}\right)}
$$

$E_{0}$ and $E_{X}^{j}\left(E_{Y}^{i}\right)$ are respectively the accelerating potential of the electrons and the critical ionization energy of the $j$-shell ( $i$-shell) of atom $X(Y)$. The number of electrons in the $j$-shell ( $i$-shell) of element $X(Y)$ is denoted $Z_{X}^{j}\left(Z_{Y}^{i}\right)$.

4.4 EVALUATION OF THE SYSTEMATIC EXTRAPOLATION ERROR. - All the terms in equation (46) will now be discussed separately. Let us first consider the ionization cross section ratio $\psi_{Y}^{i}\left(E_{0}\right) / \psi_{X}^{j}\left(E_{0}\right)$.

Whenever two K- or L- lines are considered, the ratio $Z_{Y}^{i} / Z_{X}^{j}$ equals 1 since for elements beyond $\mathrm{Ne}$ the $\mathrm{K}$ - and L-lines always contain 2 and 8 electrons. Another frequent occuring situation is that L-lines of an element $(X)$ are excited by the $\mathrm{K}$-lines of another element $(Y)$. In this case the ratio $Z_{Y}^{i} / Z_{X}^{j}$ equals $1 / 4$.

The critical excitation energy ratio $E_{X}^{j} / E_{Y}^{i}$ is certainly smaller than one since the energy of the exciting line must be higher than the energy of the excited line.

The same conclusion is true for the ratio $\ell \mathrm{n}\left(E_{0} / E_{Y}^{i}\right) / \ell \mathrm{n}\left(E_{0} / E_{X}^{j}\right)$. In other words the ionization cross section ratio $\psi_{Y}^{i}\left(E_{0}\right) / \psi_{X}^{j}\left(E_{0}\right)$ is always smaller than unity. Usually the atom mass ratio $A_{X} / A_{Y}$ is also less than 1 because the heavier element excites the other.

The fluorescence yield $\omega_{Y}^{i}$ of element $Y$, the ionization yield $r_{X}^{j}-1 / r_{X}^{j}$ of element $X$ and the mass concentration $C_{Y}$ of element $Y$ are also parameters smaller than 1 . The mass absorption 
coefficient $\mu_{Y}^{X}$ is typically of the order of $100 \mathrm{~cm}^{2} / \mathrm{g}$ and the wedge angle $\alpha$ does not exceed $90^{\circ}$. A rough estimate for the constant $K_{X Y}$ is $1 \times 10^{-4} \mathrm{~g} /\left(\mathrm{cm}^{2} \mathrm{deg}\right.$.) (see next paragraph).

Generally speaking $\lim _{T \rightarrow 0}\left(I_{X}^{\mathrm{F}} / I_{X}^{\mathrm{P}}\right)$ will always be less than $1 \%$, a value only trespassed in extreme situations.

This statement will now be checked for a $\mathrm{Cu}-50$ at\% $\mathrm{Cr}$ alloy.

From the fluorescence simulations in $\mathrm{Cu}-50$ at\% $\mathrm{Cr}$ wedges we know that [5].

$$
K_{\mathrm{CuCr}}=1.66 \times 10^{-4} \frac{\mathrm{g}}{\mathrm{cm}^{2} \mathrm{deg}}
$$

The mass absorption coefficient of the $\mathrm{Cu}-\mathrm{K}_{\alpha}$ line in a pure chromium target is extremely high and equals : $\mu_{\mathrm{Cu}}^{\mathrm{Cr}}=250 \mathrm{~cm}^{2} / \mathrm{g}$. The fluorescence yield of the $\mathrm{Cu}-\mathrm{K}$ line, $\omega_{\mathrm{Cu}}^{\mathrm{K}}$ is equal to 0.39 and the mass ratio $A_{\mathrm{Cr}} / \mathrm{A}_{\mathrm{Cu}}$ is 0.817 [12]. The ionization cross section ratio $\psi_{\mathrm{Cu}}^{\mathrm{K}}(100 \mathrm{kV}) / \psi_{\mathrm{Cr}}^{\mathrm{K}}(100 \mathrm{kV})$ is calculated with the critical ionization potentials $E_{\mathrm{Cu}}^{\mathrm{K}}$ and $E_{\mathrm{Cr}}^{\mathrm{K}}$ which are respectively equal to : $E_{\mathrm{Cu}}^{\mathrm{K}}=8.943 \mathrm{keV}$ and $E_{\mathrm{Cu}}^{\mathrm{K}}=5.957 \mathrm{keV}[13]$ :

$$
\frac{\psi_{\mathrm{Cu}}^{\mathrm{K}}(100 \mathrm{kV})}{\psi_{\mathrm{Cr}}^{\mathrm{K}}(100 \mathrm{kV})}=0.57
$$

The only remaining unknown is the ionization yield $\left(r_{\mathrm{Cr}}^{\mathrm{K}}-1\right) / r_{\mathrm{Cr}}^{\mathrm{K}}$. The absorption jump ratio $r_{\mathrm{Cr}}$ is calculated from mass absorption coefficients values in a pure chromium target. The ratio of the curve fit value above and below $E_{\mathrm{Cr}}^{\mathrm{K}}$ yields the absorption jump ratio $r_{\mathrm{Cr}}$ [5] :

$$
r_{\mathrm{Cr}}=\frac{\mu^{\mathrm{Cr}}\left(E \overrightarrow{>} E_{\mathrm{Cr}}^{\mathrm{K}}\right)}{\mu^{\mathrm{Cr}}\left(E \overleftarrow{<} E_{\mathrm{Cr}}^{\mathrm{K}}\right)}=8.58
$$

Consequently the ionization yield of the chromium K-shell equals :

$$
\frac{r_{\mathrm{Cr}}^{\mathrm{K}}-1}{r_{\mathrm{Cr}}^{\mathrm{K}}}=0.88
$$

Finally the secondary to primary emission ratio reads :

$$
\lim _{T \rightarrow 0}\left(\frac{I_{\mathrm{Cr}}^{\mathrm{F}}}{I_{\mathrm{Cr}}^{\mathrm{P}}}\right)=2.6 \times 10^{-4} \cdot \alpha
$$

where $\alpha$ the wedge angle is expressed in degrees. Note that this ratio is equal to $1 \%$ for a wedge angle of $38^{\circ}$.

From this residual ratio one can estimate the error on the final extrapolated mass concentration ratio since :

$$
\left(\frac{C_{A}}{C_{B}}\right)^{\prime}=\frac{C_{A}}{C_{B}} \frac{1+\lim _{T \rightarrow 0}\left(I_{A}^{\mathrm{F}} / I_{A}^{\mathrm{P}}\right)}{1+\lim _{T \rightarrow 0}\left(I_{B}^{\mathrm{F}} / I_{B}^{\mathrm{P}}\right)}
$$

or :

$$
\left(\frac{C_{A}}{C_{B}}\right)^{\prime}=\frac{C_{A}}{C_{B}}(1+a)
$$


where :

$$
a=\lim _{T \rightarrow 0}\left(I_{A}^{\mathrm{F}} / I_{A}^{\mathrm{P}}\right)-\lim _{T \rightarrow 0}\left(I_{B}^{\mathrm{F}} / I_{B}^{\mathrm{P}}\right)
$$

$I_{B}^{\mathrm{F}}=0$ is the worst case situation encountered in binary systems such as a $\mathrm{Cu}-50$ at $\% \mathrm{Cr}$ alloy. From (53) it is clear that :

$$
C_{A}^{\prime}=C_{A} \frac{1+a}{1+C_{A} \cdot a} \text { and } C_{A}+C_{B}=1
$$

or approximately :

$$
C_{A}^{\prime} \approx C_{A}\left(1+C_{B} \cdot a\right)
$$

The relative error on $C_{A}^{\prime}$ is the highest when $C_{B}$ approaches 1 or in other words when there is only a small fraction of fluorescing material and a large fraction of the exciting element present in the specimen. However the error on the extrapolated mass concentration $C_{A}^{\prime}$ will never exceed a which in its turn never exceeds the residual ratio $\lim _{T \rightarrow 0}\left(I_{A}^{\mathrm{F}} / I_{A}^{\mathrm{P}}\right)$.

For the $\mathrm{Cu}-50$ at\% $\mathrm{Cr}$ example the extrapolated concentrations would be :

$$
\left\{\begin{array} { l } 
{ C _ { \mathrm { Cr } } ^ { \prime } = 4 5 . 2 5 \mathrm { wt } \% } \\
{ C _ { \mathrm { Cu } } ^ { \prime } = 5 4 . 7 5 \mathrm { wt } \% }
\end{array} \text { instead of : } \left\{\begin{array}{l}
C_{\mathrm{Cr}}=45.00 \mathrm{wt} \% \\
C_{\mathrm{Cu}}=55.00 \mathrm{wt} \%
\end{array}\right.\right.
$$

The error is $0.25 \mathrm{wt} \%$ and as will be demonstrated with experimental results (see Sect. 6) the errors on the extrapolated values due to statistical fluctuations are typically of the order of 0.5 to $1.0 \mathrm{wt} \%$. Consequently the systematic error due to the linear term of the fluorescence polynomial in $T$ is smaller than the error introduced by statistical fluctuations and can therefore be neglected. An experimental confirmation of this conclusion will be given in paragraph 6 where the experimental results on a highly fluorescing system (Fe-Ni) will be discussed.

\section{Practical implementation of the method.}

5.1 INTERNAL THICKNESS MEASURE. - In section 2.2 it was already mentioned that Bremsstrahlung is not a suitable internal thickness measure especially in the limit for zero thickness. This statement will now be verified by comparing the results obtained on a $\mathrm{Cr} 85$ at\% $-\mathrm{Al}$ alloy with a Bremsstrahlung window and with a net peak integral $\left(\mathrm{Cr}-\mathrm{K}_{\alpha}\right)$.

The specimen was examined in a low background (carbon) holder at $100 \mathrm{kV}$. Ten spectra were taken at different thicknesses but all in very transparant areas and the dead time of the multi channel analyser never exceeded $35 \%$. The lifetime of the spectra is $60 \mathrm{~s}$. The uncorrected mass concentration ratios $\left(C_{\mathrm{Al}} / C_{\mathrm{Cr}}\right)^{\prime}$ are calculated with a standardless metallurgical thin film program provided by Tracor Northern, which uses calculated $k$-factors. No reliable standard specimen was available and as will be clear from the results the calculated $k$-factor is accurate enough especially for comparative experiments.

The parameterless correction procedure was carried out with 10 Bremsstrahlung windows at different energies and with the net peak integral of the $\mathrm{Cr}-\mathrm{K}_{\alpha}$ line. All the results with the Bremsstrahlung windows agree within 0.15 at $\%$ and the average extrapolated concentrations are :

$$
\left\{\begin{array}{l}
\text { at\% } \mathrm{Cr}: 85.27 \\
\text { at\% } \mathrm{Al}: 14.72
\end{array}\right.
$$


The $\mathrm{Cr}-\mathrm{K}_{\alpha}$ peak analysis yields less aluminium :

$$
\left\{\begin{array}{l}
\text { at\% } \mathrm{Cr}: 85.53 \\
\text { at\% } \mathrm{Al}: 14.46
\end{array} \pm 0.12\right.
$$

The results of the $\mathrm{Cr}-\mathrm{K}_{\alpha}$ peak analysis and the 19.5-20.0 keV Bremsstrahlung window analysis are plotted in figure 3.

From this figure can be deduced that about 40 counts in the 19.5-20.0 keV window are not originating from the specimen although a low background holder was used for the measurements.

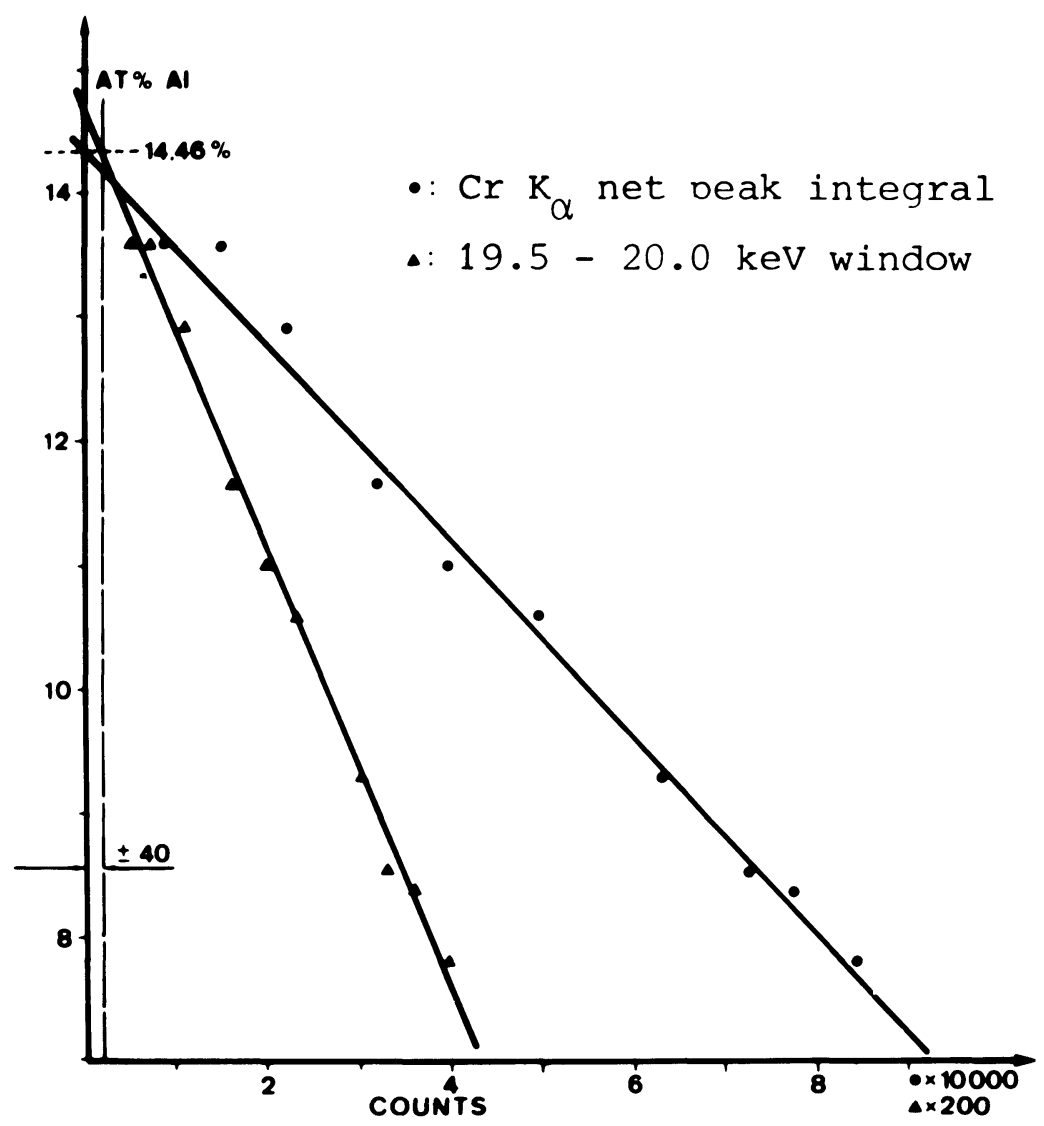

Fig. 3. - In spite of the use of a low background specimen holder, the analysis with a Bremsstrahlung window (19.5-20.0 keV) exhibits the presence of spurious X-rays (about 40 counts).

When no low background holder can be used or when for some other reason the amount of spurious $\mathrm{X}$-rays increases the difference between the two extrapolation values becomes more important. It is therefore imperative that the parameterless correction procedure should make use of characteristic radiation as internal thickness measure. This characteristic radiation can be a net peak integral or the sum of net peak integrals. 
5.2 PolynOMial Fit. - A polynomial has to be fitted through the experimental points so as to match equation (9). However, when the degree of the fitted polynomial is too high the curve fit will lose its physical significance, because of the statistical fluctuations of the experimental points. The extrapolation to net peak zero then becomes hazardous. On the other hand a systematic error can also be induced when the degree of the fit is too low. As an example the spectra for figure 3 were taken in a very thin area so that a linear curve fit seems sufficient.

Although a linear regression is sufficient in most of the cases, especially when the spectra are acquired in transparant areas of the specimen, it can be inadequate for systems with a strongly absorped element. Then $B_{1}^{A B}$ is not negligible (Eq. (9)) and a parabolic curve fit is needed. Figure 4 shows an example were the linear fit clearly fails. It is again a $\mathrm{Cr}-\mathrm{Al}$ alloy and the 9 spectra were taken till just over the transparancy limit at $100 \mathrm{kV}$. Figure 4 shows a linear fit with the 6 first points and a parabolic fit with all the points (9). Moreover a situation where a parabolic fit is inadequate was never encountered.

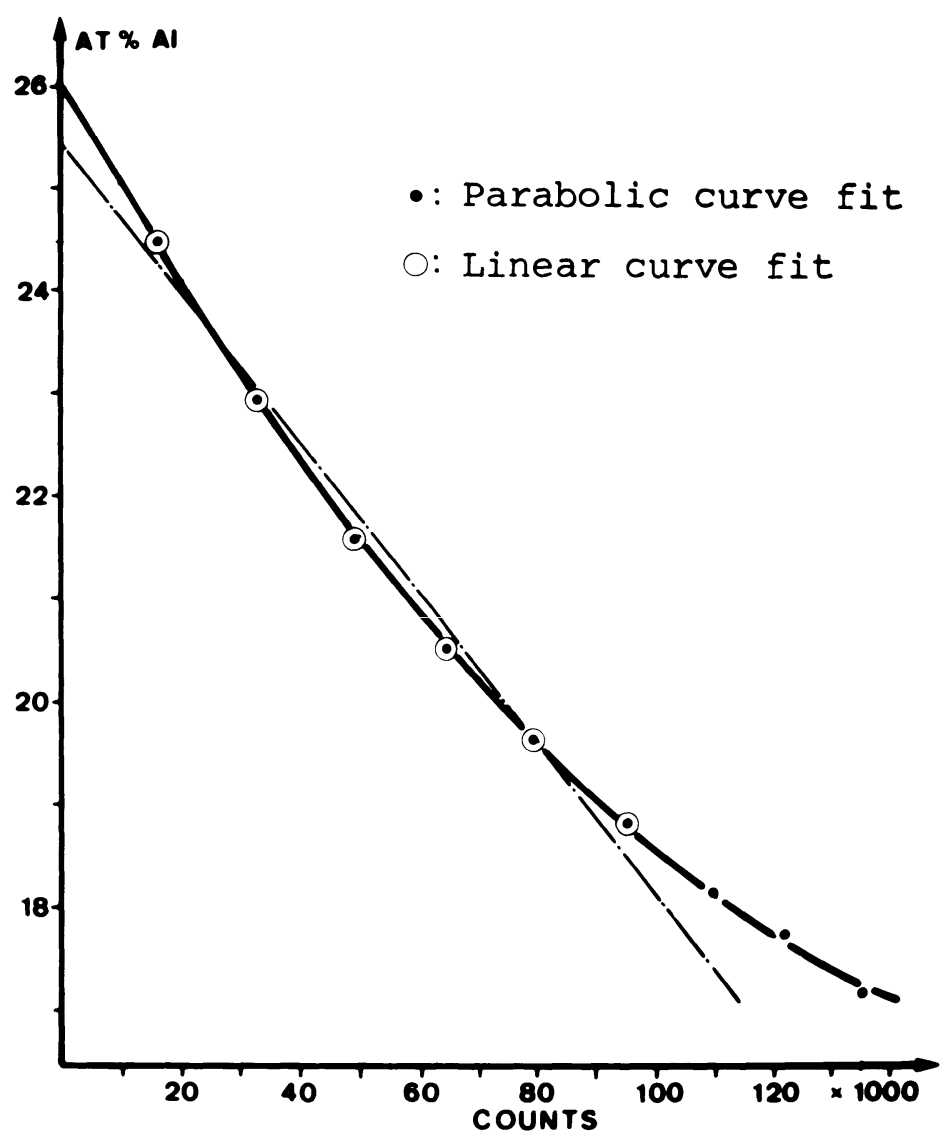

Fig. 4. - An example where a linear fit fails and a parabolic fit is needed to extrapolate the aluminium concentration of a $\mathrm{Cr}-\mathrm{Al}$ alloy.

Finally it is to the operators common sense to judge whether a linear or parabolic fit is to be used for a specific analysis. When only transparant areas are analysed one of both will always be adequate to extrapolate the corrected mass concentration ratio. 
5.3 EXTERNAL INFLUENCES. - In this paragraph some phenomena which could introduce errors in the parameterless correction method will be discussed. Some of these phenomena can be traced by the method and subsequently avoided or at least reduced.

5.3.1 Surface layers. - Surface layers can be classified in two categories ; extrinsic and intrinsic layers.

5.3.1.1 Extrinsic surface layers. - Extrinsic surface layers are layers which are deposited onto the specimen by preparation techniques (jet polishing, cleaning, ion thinning...) or by contamination build up during the microscopical investigation. These layers usually contain elements which are not present in the specimen and are therefore easily detectable. Although they increase the primary excitation volume they seem to have no significant influence on the quantitative results obtained with the parameterless correction method on condition that characteristic radiation is used as external thickness measure. This experimentally verified statement is equivalent to say that the absorption correction for the analysed elements within the extrinsic layer is negligible.

5.3.1.2 Intrinsic layers. - Intrinsic layers are layers which originally belong to the specimen. Caused or not by preparation techniques the composition of the outermost layer of the specimen can differ from the bulk composition, e.g. : selective desorption of certain elements at the surface [14]. The structure and composition of the surface can also be modified by the electron beam (radiation damage). Since these layers contain the same elements as the bulk their influence on the X-ray spectra is intrinsic and cannot be eliminated easily. However the parameterless correction method often enables to trace them since these intrinsic surface layers are approximately uniformly thick and their relative influence increases with decreasing specimen thickness. As a consequence the extrapolation curve for an element $X$ will exhibit a sudden bend for decreasing specimen thickness.

In other words, a deviation from linearity on the left hand side of the experimental curve is an indication for a surface layer. If this layer cannot be avoided, fairly good quantitative results can still be obtained by eliminating the obviously deviating experimental points. Only the measurements in the thicker regions where the relative influence of the surface layers is negligible are then considered for the extrapolations. Consequently the final results will be less accurate but still more acceptable than a quantitative analysis obtained with only one spectrum precisely taken in the thinnest region.

5.3.2 The Borrmann effect. - E.D. spectra of crystalline specimens can be affected by the orientation of the specimen in the electron beam. The main idea is that a plane wave interacting with a periodic potenial can generate a standing wave in the crystal depending on the exact impact direction. The ionization probability of an atom is proportional with the electron density $|\psi|^{2}$, which for a periodical wave function $\psi$, might differ from one atom site to another. If such a situation occurs the emission of characteristic radiation from certain elements will be favoured when compared to others and consequently the spectrum will be distorted. CHERNS and coworkers [15], demonstrated that this orientation effect can be very crucial for X-ray microanalysis when a number of Bragg reflections are strongly excited and especially for crystal thicknesses of the order of $10 \mathrm{~nm}$. For thicker targets multiple scattering will become dominant and $|\psi|^{2}$ will gradually lose its periodicity until all atom sites are equally excited.

This phenomenon is traced by the parameterless correction method in the same way as are intrinsic surface layers. The extrapolation curve will again exhibit a strange behaviour for decreasing specimen thickness. The bormann effect disturbs the spectra up to much larger thicknesses than intrinsic surface layers, however the remedy to this problem is much easier since a small tilt of the specimen away from the strongly excited Bragg reflections destroyes the responsible standing wave. 


\section{Some examples of application of the method.}

Two distinct systems will be investigated. The first testing material is a $\mathrm{Cr} 70$ at \% - Al alloy and it was chosen because of the important absorption correction due to the presence of aluminium. The second set of results concerns Fe-Ni alloys which are known to be very strongly fluorescing systems.

6.1 $\mathrm{Cr} 70$ at\% -Al. - The specimens were electropolished with a mixture of $90 \%$ methanol $\left(\mathrm{CH}_{3} \mathrm{OH}\right)$ and $10 \%$ perchloric acid $\left(\mathrm{HClO}_{4}\right)$. The spectra were acquired at $100 \mathrm{kV}$, a low background holder was used and strong Bragg reflections were carefully avoided.

A first multi spectra analysis makes use of 10 measuring sites more or less aligned perpendicular to the specimen edge and parallel to the tilt axis. After the spectra acquisitions the specimen holder was tilted from $+35^{\circ}$ to $-35^{\circ}$ so as to visualize the wedge shape geometry of the specimen. The SEM image in figure 5 shows the 9 first contamination spots in profile from which could be deduced that the wedge angle is approximately $11.5^{\circ}$.

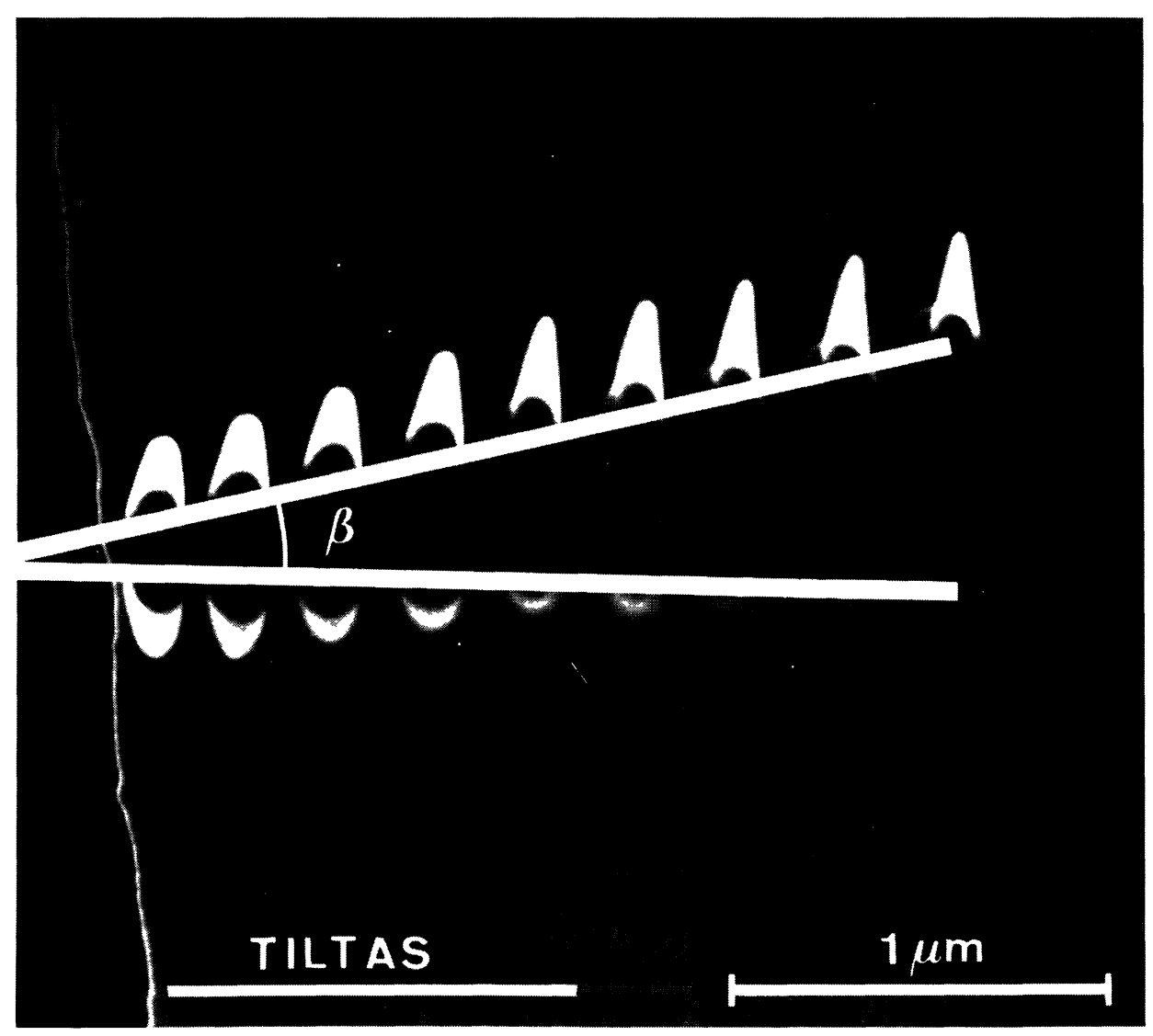

Fig. 5. - SEM image showing the contamination spots due to parameterless correction analysis. The specimen was tilted over an angle of $70^{\circ}$ and is clearly wedge shaped in the neighbourhood of the hole. 
The reason for using SEM is that it was impossible in transmission to obtain an adequate contrast and brightness of the image so as to visualize the whole thichnicss range.

The spectra of the thinnest points show small $\mathrm{Si}$ and $\mathrm{Cl}$ peaks due to contamination. When only the 9 analysis points of figure 5 are considered for the curve fit, a linear least square fit is more than adequate. When the 10th point is added it is preferable to use a parabolic fit. Two internal measures for the mass thickness are used : the $\mathrm{Cr}-\mathrm{K}_{\alpha}$ net peak and the sum of the $\mathrm{Cr}-\mathrm{K}_{\alpha}+\mathrm{Al}-\mathrm{K}_{\alpha}$ net peaks. Table I gives an overview of the results.

Table I. - Results of a 10 sites parameterless correction procedure on a $\mathrm{Cr} 70$ at\% - Al alloy. Both the $\mathrm{Cr}-\mathrm{K}_{\alpha}$ net peak and the sum of the $\mathrm{Cr}-\mathrm{K}_{\alpha}+\mathrm{Al}-\mathrm{K}_{\alpha}$ net peaks are used as an internal parameter for the mass thickness. The results with the linear fit were obtained by omitting the thickest analysis point.

\begin{tabular}{|c|c|c|c|c|c|c|}
\hline & & \multicolumn{2}{|c|}{ LINEAR : 9 POINTS } & \multicolumn{3}{|c|}{ PARABOLIC : 10 POINTS } \\
\hline $\begin{array}{c}\text { internal } \\
\text { parameter }\end{array}$ & element & at $\%$ & $w t \%$ & at\% & $w t \%$ & \\
\hline $\operatorname{CrK} \alpha$ & $\begin{array}{l}\mathrm{Al} \\
\mathrm{Cr}\end{array}$ & $\begin{array}{ll}29.47 & \\
70.53 & \pm 0.60\end{array}$ & $\begin{array}{ll}17.83 & \\
82.17 & \pm 0.51\end{array}$ & $\begin{array}{l}29.48 \\
70.52\end{array}$ & $\begin{array}{l}17.83 \\
82.17\end{array}$ & \pm 0.24 \\
\hline $\mathrm{CrK} \alpha+\mathrm{AlK} \alpha$ & $\begin{array}{l}\mathrm{Al} \\
\mathrm{Cr}\end{array}$ & $\begin{array}{l}29.55 \\
70.45\end{array}$ & $\begin{array}{ll}17.88 & \\
82.12 & \end{array}$ & $\begin{array}{ll}29.48 & \\
70.52 & \pm 0.41\end{array}$ & $\begin{array}{l}17.84 \\
82.16\end{array}$ & \pm 0.25 \\
\hline
\end{tabular}

To confirm the previous results a second analysis was carried out in an other area of the same specimen. It concerns a 12 point analysis and in each measure site spectrum of 80000 counts was acquired. Subsequently the spectra were renormalized to a lifetime of $100 \mathrm{~s}$ in order to be used for a parameterless correction. This slightly modified procedure ensures that all the spectra are statistically equivalent. The disadvantage compared to the normal procedure is the relatively long real acquisition time in the very thin areas.

The region where the 12-point analysis was carried out is thin enough so that a linear curve fit is sufficient. The results are surveyed in table II.

The results of tables I and II are in good agreement with each other and with the presumed composition Cr 70 at\% - Al. Depending on the accuracy of this presumed composition an experimental $k$-factor could now be derived, but this would only be a little larger than the calculated and used $k$-factor. The results quite obviously demonstrate that the parameterless correction procedure is a powerful way of correcting for absorption even in systems with strongly absorbed peaks.

6.2 THE Fe-Ni SYSTEM. - Iron-nickel alloys are strongly fluorescing materials. The energy of the $\mathrm{Ni}-\mathrm{K} \alpha$ peak $(7.47 \mathrm{keV})$ is sufficient to excite the K-shell of iron $\left(E_{\mathrm{K}}^{\mathrm{Fe}}=7.083 \mathrm{keV}\right)[13]$. $\mathrm{Ni}-\mathrm{K}$ radiation therefore generates secondary $\mathrm{Fe}-\mathrm{K}$ radiation with a very high yield. Consequently $\mathrm{Fe}-\mathrm{Ni}$ alloys are extremely well suited to investigate whether the parameterless correction procedure sufficiently corrects for fluorescence. 
Table II. - Results of a 12 sites parameterless correction procedure on a $\mathrm{Cr} 70$ at $\%$ - $\mathrm{Al}$ alloy. Both the $\mathrm{Cr}-\mathrm{K}_{\alpha}$ and the sum of the $\mathrm{Cr}-\mathrm{K}_{\alpha}$ and the $\mathrm{Al}-\mathrm{K}_{\alpha}$ net peaks are used as an internal parameter for the mass thickness.

\begin{tabular}{|l|c|cccc|}
\hline $\begin{array}{c}\text { internal } \\
\text { parameter }\end{array}$ & element & at\% & wt\% & \\
\hline $\mathrm{CrK} \alpha$ & $\mathrm{Al}$ & 29.29 & & 17.70 & \\
& & & \pm 0.64 & & \pm 0.56 \\
\hline \multirow{2}{*}{$\mathrm{CrK} \alpha+\mathrm{AlK} \alpha$} & $\mathrm{Al}$ & 29.36 & & 82.30 & \\
& $\mathrm{Cr}$ & 70.64 & & 82.25 & \\
\hline
\end{tabular}

6.2.1 A composition without absorption correction. - Table III is a survey of the mass absorption coefficients of the Fe- $\mathrm{K}_{\alpha}$ and Ni-K $\mathrm{K}_{\alpha}$ lines in iron and nickel targets. Thanks to the presence of an absorption edge in between the energies ui $\mathrm{Fe}-\mathrm{K}_{\alpha}(6.40 \mathrm{keV})$ and $\mathrm{Ni}-\mathrm{K}_{\alpha}(7.47 \mathrm{keV})$ in iron, a specific composition exists for which the $\mathrm{Fe}-\mathrm{K}_{\alpha}$ and $\mathrm{Ni}-\mathrm{K}_{\alpha}$ lines have an identical mass absorption coefficient. In other words no absorption correction is needed for this specific alloy, and the differences between uncorrected concentrations or concentration ratios and the real values are only due to fluorescence in the specimen. This specific composition is obtained straightforwardly (Tab. IV) and for the sake of simplicity this alloy will be referred to as : Fe 10-Ni.

Table III. - The mass absorption coefficients of the $\mathrm{Fe}-\mathrm{K}_{\alpha}$ and $\mathrm{Ni}-\mathrm{K}_{\alpha}$ lines in pure $\mathrm{Fe}$ and pure $\mathrm{Ni}$

\begin{tabular}{|c|c|c|}
\hline & in Fe & in Ni \\
\hline $\mathrm{Fe}-\mathrm{K}_{\alpha}(6.40 \mathrm{keV})$ & $70.4 \mathrm{~cm}^{2} / \mathrm{g}$ & $91.8 \mathrm{~cm}^{2} / \mathrm{g}$ \\
$\mathrm{Ni}-\mathrm{K}_{\alpha}(7.47 \mathrm{keV})$ & $367 \mathrm{~cm}^{2} / \mathrm{g}$ & $59.8 \mathrm{~cm}^{2} / \mathrm{g}$ \\
\hline
\end{tabular}

Table IV. - The composition of a Fe-Ni alloy for which no absorption correction is needed.

\begin{tabular}{|c|c|c|c|}
\hline Element & at\% & wt\% & $\mu$ \\
\hline $\mathrm{Fe}$ & $10.19 \%$ & $9.74 \%$ & \\
& & & $89.7 \mathrm{~cm}^{2} / \mathrm{g}$ \\
$\mathrm{Ni}$ & $89.81 \%$ & $90.26 \%$ & \\
\hline
\end{tabular}

Specimens of this alloy and of two other compositions, Fe 50-Ni and Fe 90-Ni were thinned electrochemically. However some Fe $10-\mathrm{Ni}$ specimens were prepared by ion thinning so as to obtain different geometries. This is achieved by placing the ion guns at different angles and specimens 
with wedge angles of 10, 40 and 60 degrees and referred to as $\alpha 10, \alpha 40$ and $\alpha 60$ were obtained. If the extrapolation value of the correction procedure is noticably influenced by the real geometry of the specimen this will certainly be observed. Two supplementary electrochemically $\mathrm{Fe} 10-\mathrm{Ni}$ specimens denoted pol 1 and pol 2 were also examined.

Two Fe-Ni alloys are used to derive an experimental $k_{\mathrm{NiFe}}$-factor : Fe $(50.00 \pm 0.07)$ wt $\%-\mathrm{Ni}$ and $\mathrm{Fe}(90.01 \pm 0.01) \mathrm{wt} \%-\mathrm{Ni}$. The result reads :

$$
k_{\mathrm{NiFe}}=0.84 \pm 0.01
$$

The experiments with the Fe $10-\mathrm{Ni}$ specimens are carried out with this $k$-factor.

6.2.2 Experimental results for the Fe 10-Ni alloy. - The five Fe 10-Ni specimens are examined in a single tilt low background holder at $100 \mathrm{kV}$ and all the spectra are taken in electron transparant areas. Since the specimens have approximately the composition given in table IV, no absorption correction is needed even for the thickest regions.

On each specimen 3 independent parameterless analysis are carried out and the results are surveyed in table $\mathrm{V}$.

Table V. - Compositions measured with the parameterless correction procedure (in at\%) on different Fe 10 - Ni specimens.

\begin{tabular}{|c|c|c|c|}
\hline at\% & 1st ANALYSIS & 2nd ANALYSIS & 3rd ANALYSIS \\
\hline$\alpha 10 \begin{array}{ll} & \mathrm{Fe} \\
& \mathrm{Ni}\end{array}$ & $\begin{array}{r}9.48 \\
90.52\end{array}$ & $\begin{array}{c}8.88 \\
\quad \pm 1.33 \\
91.12\end{array}$ & $\begin{array}{c}9.45 \\
90.55\end{array}$ \\
\hline $\begin{array}{ll} & \mathrm{Fe} \\
\alpha 40 & \\
& \mathrm{Ni}\end{array}$ & $\begin{array}{ll}9.58 & \\
90.42 & \pm 0.67\end{array}$ & $\begin{array}{l}10.10 \\
89.90\end{array}$ & $\begin{array}{c}9.01 \\
90.99\end{array}$ \\
\hline $\begin{array}{ll} & \mathrm{Fe} \\
& \\
& \mathrm{Ni}\end{array}$ & $\begin{array}{c}9.72 \\
90.28\end{array}$ & $\begin{array}{l}10.03 \\
89.97\end{array}$ & $\begin{array}{r}9.85 \\
90.15\end{array}$ \\
\hline 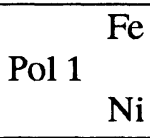 & $\begin{array}{l}10.30 \\
89.70\end{array}$ & $\begin{array}{c}9.26 \\
90.74\end{array}$ & $\begin{array}{ll}9.83 & \\
90.17 & \pm 1.18\end{array}$ \\
\hline Pol $2{ }^{\mathrm{Fe}}$ & $\begin{array}{l}9.90 \\
90.10\end{array}$ & $\begin{array}{l}9.88 \\
90.12 \\
\quad \pm 1.35\end{array}$ & $\begin{array}{c}9.72 \\
90.28\end{array}$ \\
\hline
\end{tabular}

The relatively large errors are due to the fact that here fluorescence is the only phenomenon which influences the uncorrected concentrations (or concentration ratios). In other words the uncorrected values will be somewhat influenced by the local changes in the specimen geometry. Consequently the errors on the extrapolation values will be larger than for specimens where absorption dominates.

No distinction can be made between the different specimens and all the results are consistent with the presumed composition (Tab. IV). The weighted average of the results of table V can be considered as the most accurate estimation of the final composition and is listed in table VI. 
for. Beside this unquestionable advantage and also in contrast with the classical approaches, the described correction procedure provides the final results with an accuracy figure. This statistical error, which typically lies between 0.2 and 1.0 at-wt $\%$ easily permits to distinguish between well and badly performed experiments, although it does not account for the systematic error introduced by the use of a wrong $k$-factor. About this $k$-factor problem it should be mentioned that Carpenter et al. (1988) [17] used the method precisely to determine $k$-factors provided with accuracy estimates.

Furthermore, mass thickness dependent artefacts such as surface layers or a crystallographic orientation effect (the Bormann effect which can become very important in the very thin sections of the specimen are detected by the method.

Finally the main achievement of this correction procedure probably remains the fact that no external parameters such as thickness and density of the specimen, mass absorption coefficients, fluorescence yields etc. ... are needed. This guarantees a fast and non speculative processing of the acquired spectral data.

\section{References}

[1] TIXIER R., Ph. D. Thesis, IRSID (1972).

[2] Philibert J. and TIXIER R., Physical Aspects of Electron Microscopy and Microbeam analysis (J. Wiley and Sons) 1975, p. 333.

[3] Nockolds C., NaSir M. J., Cliff G. and Lorimer G. W., Inst. Phys. Conf. Ser. 52 (1980) 417.

[4] Van CAPPEllen E., Deblieck R., Van LanduYt J. and AdAMS F., J. Trace Microprobe Techn. 2 (1984) 139.

[5] VAN CAPPELlen E., DebliECK R. and VAN DYCK D., to be submitted.

[6] VAN CAPPEllen E., VAN Dyck D., VAN LANDUYT J. and ADAMS F., J. Microsc. Spectrosc. Electron. 8 (1983) $16 a$.

[7] Van Cappellen E., Van Dyck D., Van LanduYt J. and Adams F., J. Phys. Colloq. France 45 (1984) C2-411.

[8] VAN CAPPELlen E., proc. ICXOM 11, Eds. J. D. Brown and R. H. Packwood (London, Canada) 1986, p. 409.

[9] Cliff G. and LoRimer G. W., J. Microsc. 103 (1975) 203.

[10] HALl T. A., J. Microsc. 117 (1979) 145.

[11] Philibert J. and Tixier R., Brit. J. Appl. Phys. (J. Phys. D.) Ser. 21 (1986) 685.

[12] Handbook of Chemistry and Physics, 56th Ed. 1975-76 (CRC Press) 1975.

[13] Atomic Data (Academic Press) Vol. 3, $\mathrm{n}^{\circ} 1$ (1971).

[14] DoIG P. and FLEWITT P. E., J. Microsc. 110 (1977) 107.

[15] CHERNS D., HowIE A. and JACOBS M. H., Z. Naturforsch. 28a (1973) 365.

[16] Van Cappellen E., Van Landuyt J. and Adams F., Anal. Chim. Acta 195 (1987) 257.

[17] CARPENTER G. J. C. and WOO O. T., EMSA Bulletin 18 (1988) 57. 